RESEARCH REPORT

\title{
PRESENCE OF DYSPHONIA IN INDIVIDUALS WITH TOURETTE'S SYNDROME
}

\begin{abstract}
Professionals working in the field of voice disorders have come to recognize the variability of vocal qualities in different populations. While voice disorders can be of organic, neurogenic, or functional etiology, concomitant disorders often directly impact the vocal features. Such a disorder is Tourette's Syndrome which is an impairment characterized by motor and vocal tics. This study examined the prevalence of voice disorders in individuals with Tourette's Syndrome. Research was conducted over the duration of two years with clientele aged 15;2 to 26;5. Participants were receiving continuous treatment from a team of neurologist and psychologist at private clinics situated around a suburban area. Two case studies consisting of 1-2 individuals were also closely examined to further distinguish the types of voice disorders present given the severity of motor and vocal tics. Instrumental and perceptual analysis was obtained to accurately diagnose the voice disorder. Given the sample of participants, presence of spasmodic dysphonia and falsetto were noted. Results of this study indicates a strong presence of dysphonia in individuals with Tourette's Syndrome, particularly spasmodic dysphonia and falsetto. Additionally, dysphonic vocal qualities were irrelevant to the existence of vocal tics. Further research with this population is mandated to determine assessment and treatment strategies.
\end{abstract}

\section{Keywords}

Tourette's Syndrome, voice disorder, dysphonia, spasmodic dysphonia, falsetto, OCD, Botox, psychosocial factors

\section{Humaira Hussain}

Clinical speech and Language Pathologist

Institute of Physical Medicine and Rehabilitation

Dow University of Health Sciences Humairahussain.slp@gmail.com

[Hussain H. Presence of Dysphonia in Individuals with Tourette's Syndrome. Pak. j. rehabil; 2015;4(1):42-47] 


\section{INTRODUCTION}

Overtime public awareness and scientific inquiry in various disorders related to voice has increased. Voice disorders once used to be an area with little expertise and research; however, professionals such as Voice Therapist and Speech and Language Pathologist have overtime gained more insight with this dysphonic group and are now providing services to individuals with issues related to voice. While there are several types of issues related to voice ranging from functional misuse, to organic problems; a particular group of voice disorder related to neurogenic etiologies calls for enormous research and interest by professionals in this field. Neurogenic and functional voice disorders are of particular interest due to their co-existence with many motor disorders such as Tourette syndrome. The presence of dysphonia in individuals with Tourette's syndrome is prevalent, however due to the complex nature of this disorder, the dysphonia is given little attention and intervention.

\section{Overview of Voice Production}

The mechanisms involved in voice production are essential to understand while speaking of voice disorders. Normal voice production is interplay of four main systems: respiration, phonation, resonance, and articulation. Voice is produced by the vibration of the vocal folds which are a pair of muscle that stretch across the top of the trachea. They are contained within a larger structure called the larynx. The primary function of the larynx is to protect the airway during a swallow by keeping all foods and fluids out of the airway'. Its secondary function however, is voice production. The larynx contains the important structures needed for voice production including the vocal folds which are covered by the cartilages of the larynx such as: thyroid and arytenoid which aid in pulling towards and against the vocal folds to aid in vibration and production of voice?

In order for voice to be produced, it must use the respiratory system for air flow and pressure. Air is pushed against the space in between the vocal folds called, the glottis; allowing the vocal folds to vibrate. Briefly, the stronger the breathe support, the more air an individual will have to sustain voice and project it to their listeners ${ }^{2}$.

Moreover, voice production involves resonance which is the modification of voice by the cavities. Nasal and oral cavities modify sound to determine tone and quality of your voice. It also helps to limit harshness and strain in your voice by balancing the use of all resonators ${ }^{3}$. Oral cavity acts as a medium which changes the sounds by the size and shape of the articulators: mouth, lips, teeth, tongue, jaw, hard, and soft palate ${ }^{4}$. These articulators provide the muscle movement needed for speech to be produced while resonance helps modify and shape the voice perception in speech ${ }^{5}$.

Recent studies have shown that voice disorders in movement disorders such as, Tourette's syndrome is an emerging concept. Further, a thorough discussion of the movement disorder and its association to two types of voice disorders: neurogenic and functional, will be provided 6 .

\section{Gilles de la Tourette Syndrome}

Gilles de la Tourette syndrome or Tourette syndrome is chronic motor and vocal tic disorder. The age of onset for Tourette syndrome is relatively 5-7 years of age with vocal tics being produced later in life around 11-12 years of age. While it is considered psychological, recent research has shown that Tourette syndrome can be a biological disorder involving genetic abnormalities and familial predisposition. It is characterized by motor and vocal tics which vary in severity and frequency of occurrence. These tics are often aggravated by anxiety and stress ${ }^{7}$.

Previous research has also revealed a presence of co-morbid deficits such as attention deficit disorder (ADD), language and learning disorders, mood disorders, anxiety disorders and obsessive compulsive disorders $(O C D)^{6}$. Research suggests that these co-morbidities are often more troublesome and draw more attention than the actual tics ${ }^{6}$. Furthermore, vocal and phonic tics often indicate a presence of dysfluency ${ }^{8}$. These tics can occur during the beginning of speech or during speech pauses. A high number of individuals with Tourette syndrome have reported to be stutterers as a child. In fact, most are diagnosed as being stutterers before the diagnosis of TS'. Tics on the other hand are the dominant characteristic of Tourette syndrome. It is also the reason this syndrome is frequently referred to as Tics Disorder. They are characterized as sudden, repetitive, and stereotyped motor movements or phonic productions. In other words, they are involuntary muscle movements which are produced more often in times of stress and fatigue. Motor tics are the most commonly and initially appearing. These include movements such as: eye blinking, grimacing, nose twitching, jaw snaps, tooth clicking, finger flexing, and protrusion of the tongue, muscle tensing, shoulder shrugging, heard turning, or rapid jerking of any part of the body ${ }^{3}$.

Phonic tics are later appearing around 8-15 years of age. Phonic tics are fast, sounds or noises such as throat clearing, coughing, sniffling, grunting and other meaningless sounds. Phonic tics involve abnormal sounds and noise. On the contrary, vocal tics consist of linguistically meaningful utterances such as syllables, words, and phrases which involve the use of the vocal folds. Often times, sudden change in rate, rhythm and volume are observed in vocal tics ${ }^{10}$. 
There are two main types of vocal tics present in individuals with Tourette's syndrome. The first characteristic of a vocal tic involves sudden linguistic utterances such as echolalia and coprolalia" ${ }^{11}$. Coprolalia is a term used to define socially inappropriate utterances or words. Individuals with Tourette's syndrome have defined a need to complete a tic in a certain way or a certain number of times to relieve an urge, referred to as, a premonitory urge. Echolalia simply refers to repeating phrase or words spoken by someone else ${ }^{12}$.

The second type of characteristic of a vocal tic involves a sudden change in intonation and prosody. Individuals have reported to experience a sudden change in volume due to excitement or anxiety ${ }^{13}$. Some severe cases of Tourette syndrome also have reported to use different accents. Individuals with Tourette syndrome are also likely to have various psychological disorders due to which they use their voice to speak in a different register. They imitate a character's voice as if it were theirs. They also exchange dialogues using different registers ${ }^{14}$.

\section{Dysphonia in Individuals with Tourette's Syndrome}

It has been evident that voice problems do exist in individuals with Tourette syndrome. Unfortunately, due to the complex nature of Tourette syndrome, voice disorders are not given priority; rather associated disorders such as ADD/ADHD, and behavior disorders take priority in an individual's therapy ${ }^{15}$. A retroperspective study was conducted over duration of two years to establish a direct correlation between voice disorders in individuals with Tourette's syndrome. Over the past therapeutic interventions, researchers have tried to eliminate associated disorders through rehabilitation, and have used pharmacological approach to control tics. They have believed that vocal dysphonia is managed once these factors have been controlled ${ }^{12}$. This study suggested that due to the sudden changes in intonation and prosody and the effect of hyper-functionality of the vocal folds; individuals with Tourette syndrome often have voice disorders that require individualized voice therapy. Moreover, according to the data collected, it is suggested that voice disorders may not only be a cause of Tourette's syndrome, rather, it is an additional associated disorder determined by the nature and severity of the vocal tics in an individual.

\section{METHODOLOGY}

A longitudinal study over the duration of two years was completed to identify presence of voice disorders in individuals, aged 15;2-26;5 with Tourette's syndrome. Data was collected using multiple groups from a mixed socio-economic culture. Three private clinics were identified where treatment was being provided for Tourette's syndrome by a team of neurologist and psychologist. Given consent of the patient, or patient's parents, if underage, video recordings were obtained to identify presence and kind of dysphonia in individuals with Tourette's syndrome. None of the participants were previously diagnosed with voice disorder and did not ever receive voice/speech therapy. After data collection, a single-subject case study was completed to assess for validity and reliability of the research.

Neurogenic Voice Disorders: Spasmodic Dysphonia Voice problems can be related to an organic etiology, when there is a structural deficit; neurogenic etiology, with a deficit in the nervous system; or a functional etiology, when the cause is primarily vocal misuse or overuse ${ }^{16}$. While much research has been done to identify various voice disorders related to all three etiologies, nervous system involvement in voice issues is still an area to explore further.

The larynx and resonators involved in voice production are also innervated by the central and peripheral nervous systems. These systems work together to coordinate the laryngeal movements such as elevation of the larynx. Additionally, cranial and spinal nerves innervate the structures involved in voicing and provide vascularization. Spasmodic dysphonia is one of the neurogenic voice disorder caused by a dysfunction in the nervous system. In this particular group, the vocal folds vibrate normally; however intermittent spams occur during speech. There are two types of spasmodic dysphonia; adductor spasmodic dysphonia and abductor spasmodic dysphonia' ${ }^{15}$.

Adductor spasmodic dysphonia is the most common type of spasmodic dysphonia in which the thyroarytenoid muscle contracts suddenly as if in a muscle spasm. This causes the vocal folds to suddenly squeeze together very tightly, resulting in a strangled or breaking voice ${ }^{17,18}$. Abductor spasmodic dysphonia is seen less often and is caused by the sudden contraction of the posterior cricoarytenoid muscle causing the vocal folds to pull apart suddenly and results in a breathy voice'.

Spasmodic dysphonia is also characterized by a breakdown in multiple cranial nerves. Therefore, when a body is stressed, the nerves break down causing uncontrolled spasmodic muscle contractions, referred to as, dystonia. Dystonia is a neurological dysfunction of the motor movements. They may be focal, involving a few muscles; segmental, involving a group of muscles; or general, involving larger areas of the body ${ }^{19}$. Spasmodic dysphonia would be classified as focal dystonia involving the larynx. Dystonia can be initiated by trauma, genetic link, and show a variety of brain abnormalities ${ }^{20}$. Individuals who have tremors often have spasmodic dysphonia. Likewise, individuals with motor disorders and psychogenic problems such as Tourette's syndrome are also believed to present vocal dystonia, similar to spasmodic dysphonia ${ }^{5}$. 


\section{Spasmodic Dysphonia in Individuals with Tourette Syndrome}

Individuals with psychogenic problems show signs of hyperfunctional dysphonia, triggered by high stress and fatigue. In other words, dysphonic voice is produced when the individuals are stressed. The same incidence of high stress also triggers vocal tics in individuals with Tourette's syndrome. On the other hand symptoms of vocal dysphonia are persistent since time of onset in individuals with spasmodic dysphonia. Therefore it can be concluded that dysphonia is only present in times of stress and fatigue in individuals with Tourette's syndrome, whereas, in individuals diagnosed only with spasmodic dysphonia, the voice quality persevered $^{5}$. The voice is perceived similarly in those who only have a dysphonic voice as an associated deficit of Tourette syndrome and those who have adductor spasmodic dysphonia. There is a loss of projection in the voice along with a hoarse and harsh quality due to the hyper-functionality of the vocal folds.

Evidence was also found indicating using instrumental analysis to support this hypothesis. Laryngoscopy revealed similar vocal fold functions during spasmodic dysphonia, also seen in individuals with Tourette syndrome. Laryngeal electromyography (LEMG) is a diagnostic tool used for motor disorders. It helps to establish the cause of laryngeal immobility or dysfunction and also helps in locating the correct place for botulinum toxin, a common treatment for laryngeal hyperfunction in individuals with motor disorders such as Tourette syndrome ${ }^{14,21}$. It was noted that the vocal folds have the same appearance and activity. LEMG visualized similar vocal bursts activities during phonation; however, at rest, both groups represented normal functioning of the vocal folds. This similarity was driven from individvals with Tourette syndrome who were either in a vocal tic or immediately following a vocal tic. In brief, the muscle activity was similar to that of adductor spasmodic dysphonia during and following a vocal tic 22 .

Individuals with adductor spasmodic dysphonia are perceived with a creaky, choked, strained voice. They also present hyperadduction of the vocal folds during phonation. Findings are consistent across many researchers, suggesting minimal changes in vocal fold patterns of movement and appearance in individuals with spasmodic dysphonia during times of stress $^{23}$. Similar laryngoscopic results were revealed in older individuals with Tourette syndrome. Additional research on sensory deviations has revealed reflexes characterized by laryngeal adduction in individuals with Tourette syndrome. Similar to individuals with spasmodic dysphonia, individuals with Tourette syndrome who present a vocal dystonia are unable to control their urge to engage in a muscle spasm related to voice ${ }^{3}$.
Case-Study: Adductor Spasmodic Dysphonia in Individuals with Tourette Syndrome

A brief single subject case study was conducted in addition to the longitudinal study in order to verify the validity of the data collected. The subject was a 22-year old man, diagnosed with Tourette syndrome early in his life. He presented phonic tics such as: throat clearing and grunting and also had common motor tics such as shoulder and hand jerks. He received a moderate dose of Haloperidol to control his motor and vocal tics. At around the age of 19, the patient noticed a strained and creaking voice. At the time of a voice evaluation, the patient had no motor or vocal tics and had discontinued medication several years ago.

The patient was diagnosed with spasmodic dysphonia due to a hoarse, strained voice of low volume and high pitch. Observers noticed tense jaw and larynx during phonation. He was unable to project his voice with power and proper loudness. Additionally he experienced secondary symptoms such as, tightening of the mouth and throat during speech production. These symptoms are consistent with those of spasmodic dysphonia. However, it was unusual for this man to be diagnosed with spasmodic dysphonia because of his age. Usually individuals with spasmodic dysphonia are at a mean age of 44 years.

Due to a lack of pre-existing research in this area, it is unclear whether spasmodic dysphonia was related to the use of neurogenic drug, Haloperidol, or if it was an underlying idiopathic condition. It can however be said that vocal symptoms of spasmodic dysphonia were present in this patient in coexistence to Tourette syndrome. Patient presented with hyperfunctional use of the vocal folds during a vocal tic. It is suggested that the sudden urge to complete and engage in a tic is causing the hyperfunctional adduction of the vocal folds.

\section{Functional Voice Disorders: Mutational Falsetto}

Functional voice disorders include psychogenic voice disorders, in which the voice problem is directly related to psychological trauma or any psychological conflicts that may hinder normal voice production ${ }^{24}$. They also include muscle tension dysphonia, which are a group of functional voice disorders related to how the voice is misused and overused. Mutational falsetto is a type of a psychogenic voice disorder in which the vocal register of the individual does not match her age, gender, and physical appearance. Mutational falsetto consists of a high-register voice and is commonly seen in male adolescents who cannot decrease their pitch. Voice therapy aims to lower the pitch and create a vocal register appropriate for their age and gender ${ }^{25}$. 
Case Study: Mutational Falsetto in individuals with Tourette Syndrome

A second single subject case study on two individuals with diagnosis of Tourette syndrome was completed. In the first subject, a falsetto register was noticed from a 14-year old boy. Several vocal attributes were present including: throat clearing, persistent echolalia, and mumbling to oneself. A change in intonation and prosody was also noted due to frequently occurring vocal outbursts. Periodic pitch breaks were present in a low register associated with mutational falsetto. The voice was perceived as broken with repetitive and spasmodic throat clearing. Such behaviors increased with increased anxiety. It is important to note that despite administration of haloperidol to reduce motor tics, the vocal falsetto was unchanged. Therefore, it can be suggested that the falsetto was not related to any pharmacological side effect; rather it was an associated disorder.

The second individual observed was a man diagnosed with Tourette syndrome, mutational falsetto, OCD, and separation anxiety disorder. This subject presented various vocal changes in intonation and prosody. He engaged in excessive talking back and forth to himself in different intonation, mumbling, and mimicking in various vocal registers. As per previously done evidence-based research, consistently using a different vocal register to facilitate speech causes voice disorders because the individval is not speaking at their comfortable and optimal pitch, causing strain and fatigue on the vocal folds ${ }^{11}$. Furthermore, a strong association of the presence of falsetto in individuals with Tourette's Syndrome is indicated.

\section{CONCLUSION}

While there seems to be an emerging interest in the presence of adductor spasmodic dysphonia and mutational falsetto in individuals with Tourette's syndrome; a lack of extensive research leaves clinicians with fewer tools to apply in treatment for voice disorders in this population. This initial research aims to suggest a strong association between the two and establishing the presence of two types of voice disorders: spasmodic dysphonia and mutational falsetto in individuals with Tourette syndrome. At this point, data gathered from this research cannot be generalized to a vast population, however this study can be used as a pilot experiment to gain further insight into this topic.

\section{ACKNOWLEDGEMENT}

A significant portion of this research was made possible due to contributions by Adam Group of Voice Therapists and participating Zylan Clinics.

\section{REFERENCES}

[1] Boone, D., McFarlane, S., Von Berg, S., \& Zraick, $R$. The voice and voice therapy. 8th ed. Boston: Allyn and Bacon; 2010

[2] Colton, R., Casper, J., \& Leonard, R. Understanding voice problems: A physiological perspective for diagnosis and treatment. 3rd ed. Baltimore: Lippincott Williams and Wilkins; 2006.

[3] Kimaid P, Quagliato E, Crespo A, Wolf A, Viana $M$, Resende L. Laryngeal electromyography in movement disorders: preliminary data. Arq Neuro-Psiquiatr. 2004;62(3a):741-744.

[4] Cohen, D., Brunn, R., \& Leckman, J. Tourette's syndrome and tic disorder. (1st ed., pp. 4-10, 342-343). Canada: John Wiley \& Sons Inc; 1988

[5] Chowdhury U, Christie D. Tourette syndrome and tics. Current Paediatrics. 2003;13(1):42-46.

[6] Grant I, Adams K. Neuropsychological assessment of neuropsychiatric and neuromedical disorders. New York, NY: Oxford University Press; 2009.

[7] Jankovic J. Botulinum toxin in the treatment of dystonic tics. Movement Disorders. 1994:9(3):347-349.

[8] International symposium on Giles de la Tourette syndrome. Biochemical Pharmacology. $1981 ; 30(7): 815$.

[9] Kwak, C., \& Jankovic, J. (2002). Tourette syndrome: The neurology of a tic.American Speech and Hearing Association: The Leader, Retrieved from http://www.asha.org/Publications/leader/2002/020806/020806a.htm

[10] Tourette syndrome: Fact sheet. In (2011).National Institute of Neurological Disorders and Stroke (11-2163 ed.). Bathesda: Retrieved from http://www.ninds.nih.gov/disorders/tourette/detail_tourette.htm

[1 1] St-John W. Historical Perspective on 'Electromyography of intrinsic laryngeal muscles' by Fritz Buchthal. Experimental Physiology. 2008;93(2):210-212.

[12] Tetnowski, J., \& Donaher, J. (2003).Disfluency associated with tourette's syndrome: Two case studies. Informally published manuscript, Communications Disoders, Minnesota State University, Mankota, USA. , Available from Illiad. (id=')Retrieved from http://www.mnsu.edu/comdis/isad6/papers/donaher6.html

[13] Higgins, M., \& Netsell, R. (1994). Aerodynamic and electroglottographic measures of normal voice production. Journal of Speech and Hearing Research, 37, 38-45. Retrieved from http://jslhr.asha.org/cgi/content/abstract/37/1/38

[14] Leckman J, de Lotbiniere A, Marek K, Gracco C, Scahill L, Cohen D. Severe disturbances in speech, swallowing, and gait following stereotactic infrathalamic lesions in Gilles de la Tourette's syndrome. Neurology. 1993;43(5):890-890. 
[15] Lotia M, Jankovic J. Botulinum Toxin for the Treatment of Tremor and Tics. Seminars in Neurology. 2016;36(01):054-063.

[16] Hartman D, Abbs J, Vishwanat B. Clinical Investigations of Adductor Spastic Dysphonia. Ann Otol Rhinol Laryngol. 1988;97(3):247-252.

[17] Ludlow C. Stuttering: dysfunction in a complex and dynamic system. Brain. 2000;123(10):1983-1984.

[18] Van Borsel, J., \& Vanryckeghem, M. (2000). Dysfluency and phonic tics in tourette syndrome: A case report.Journal of Communications Disorders, 33, 227-240.

[19] Ludlow, C., \& Louck, T. (2003). Stuttering: A dynamic motor control disorder. National Instituite of Neurological Disorders and Stroke,28, 273-295.

[20] Robertson, M. (2011). Gilles de la tourette syndrome: The complexities of phenotype and treatment. British Journal of Hospital Medicine, 72(2), 100-106.

[21] Sataloff, R., Mandel, S., Mann, E., \& Ludlow, C.
(2003). Laryngeal electromyography: An evidence-based review. American Association of Electrodiagnostic Medicine, 28(6), 767-772. Retrieved from http://www.ncbi.nlm.nih.gov/pubmed/14639595

[22] Kwak, C., Hanna, P., \& Jankovic, J. (2000). Botulinum toxin in treatment of tics. Archives of Neurology, 57(8), 1190-1193. Retrieved from http://archneur.ama-assn.org/cgi/content/full/57/8/1190

[23] Lang, A., \& Marsden, D. (1991). Spasmodic dysphonia in gilles de la tourette's disease. Journal of Clinical Psychology, 47(2), 51.

[24] Kerbeshian, J., \& Burd, L. (1988). Tourette syndrome and mutational falsetto. Neuroscience and Biobehavioral Reviews, 12, 271-273.

[25] Elstner K, Selai C, Trimble M, Robertson M. Quality of Life (QOL) of patients with Gilles de la Tourette's syndrome. Acta Psychiatrica Scandinavica. 2008;103(1):52-59. 